\title{
Parametrizations of the $\gamma^{*} N \rightarrow \Delta(1232)$ quadrupole form factors and Siegert's theorem
}

\author{
G. Ramalho \\ International Institute of Physics, Federal University of Rio Grande do Norte, \\ Campus Universitário - Lagoa Nova, CP. 1613, Natal-RN, 59078-970, Brazil
}

(Dated: November 11, 2018)

\begin{abstract}
The large $N_{c}$ limit provides relations that can be used to calculate the $\gamma^{*} N \rightarrow \Delta(1232)$ quadrupole form factors at low and intermediate $Q^{2}$ under the assumption of the pion cloud dominance. There are two limitations in those parametrizations. First, the parametrization of the Coulomb quadrupole form factor underestimate the low $Q^{2}$ data. Second, when extrapolated for the timelike region, the form factors violate Siegert's theorem by terms of the order $1 / N_{c}^{2}$. We propose here corrections to the parametrization of the electric quadrupole form factor, which violate Siegert's theorem only by terms of the order $1 / N_{c}^{4}$. Combining the improved large $N_{c}$ pion cloud parametrizations with the valence quark contributions based on a covariant quark model for the quadrupole transition form factors, we obtain an extrapolation to the timelike region consistent with Siegert's theorem, and accomplish also a very good description of the data.
\end{abstract}

\section{INTRODUCTION}

The $\gamma^{*} N \rightarrow \Delta(1232)$ transition is characterized by the dominant dipole form factor $\left(G_{M}\right)$ and two sub-leading quadrupole form factors: the electric $\left(G_{E}\right)$ and the Coulomb $\left(G_{C}\right)$ form factors [1-4]. The nonzero results for the quadrupole form factors are a consequence of the deviation of the $\Delta(1232)$ from a spherical shape [2, [5, 6] $]$.

Calculations based on the limit of a large number of colors $\left(N_{c}\right)$ and $S U(6)$ quark models with symmetry breaking suggest that, in the low $Q^{2}$ region the $\gamma^{*} N \rightarrow \Delta(1232)$ quadrupole form factors are dominated by pion cloud effects [2, 7 12]. Estimates based exclusively on valence quarks underestimate the data by about an order of magnitude [3, 4, 13 15]. Although small comparative to the leading order pion cloud contributions, the valence quark contributions can nevertheless help to improve the description of the data [15 17]. The large $N_{c}$ parametrizations of the $\gamma^{*} N \rightarrow \Delta(1232)$ electric and Coulomb quadrupole form factors have, however, a problem: they are in conflict with Siegert's theorem [18 21].

In the form factors representation, Siegert's theorem is expressed by the identity at the pseudo-threshold: $G_{E}=\frac{M_{\Delta}-M}{2 M_{\Delta}} G_{C}[1,19,20]$ ( $M$ and $M_{\Delta}$ are respectively the nucleon and the $\Delta$ masses). The pseudo-threshold is the limit where the magnitude of the photon threemomentum, $|\mathbf{q}|$, vanishes, and $Q^{2}=Q_{p t}^{2}=-\left(M_{\Delta}-M\right)^{2}$. A test for the validity of Siegert's theorem is the value of

$$
\mathcal{R}_{p t}=G_{E}\left(Q_{p t}^{2}\right)-\kappa G_{C}\left(Q_{p t}^{2}\right)
$$

where

$$
\kappa=\frac{M_{\Delta}-M}{2 M_{\Delta}}
$$

When $\mathcal{R}_{p t}=0$, Siegert's theorem is verified. When
$\mathcal{R}_{p t} \neq 0$, the form factors are inconsistent with Siegert's theorem.

The combination of $S U(6)$ quark models with twobody exchange currents and the large $N_{c}$ limit provides a connection between the neutron charge distribution and the $\gamma^{*} N \rightarrow \Delta(1232)$ quadrupole form factors [10, 11]. In an exact $S U(6)$ quark model, the neutron electric form factor vanishes and the electric and Coulomb quadrupole moments of the $\gamma^{*} N \rightarrow \Delta(1232)$ transition are both zero. When the $S U(6)$ symmetry is broken we can relate the quadrupole moments with the neutron square charge radius $r_{n}^{2}$ [7-12]. We can then conclude that the $S U(6)$ symmetry breaking induces an asymmetric distribution of charge in the nucleon, which generates nonzero results for the neutron electric form factor $G_{E n}$, and for the $\gamma^{*} N \rightarrow \Delta(1232)$ quadrupole transition form factors, $G_{E}$ and $G_{C}[8,[9,11]$.

Moreover, based on the low $Q^{2}$ expansion of the neutron electric form factor, $G_{E n} \simeq-\frac{1}{6} r_{n}^{2} Q^{2}$, we can extrapolate the $Q^{2}$ dependence of the quadrupole form factors to [7, 9 -11]

$$
\begin{aligned}
G_{E}\left(Q^{2}\right) & =\left(\frac{M}{M_{\Delta}}\right)^{3 / 2} \frac{M_{\Delta}^{2}-M^{2}}{2 \sqrt{2}} \tilde{G}_{E n}\left(Q^{2}\right) \\
G_{C}\left(Q^{2}\right) & =\left(\frac{M}{M_{\Delta}}\right)^{1 / 2} \sqrt{2} M M_{\Delta} \tilde{G}_{E n}\left(Q^{2}\right),
\end{aligned}
$$

where $\tilde{G}_{E n}=G_{E n} / Q^{2}$. Hereafter we refer those results as large $N_{c}$ relations, since they can be derived exclusively in the large $N_{c}$ limit [7].

In this work we present improved large $N_{c}$ pion cloud parametrizations for the quadrupole form factors in order to obtain a parametrization consistent simultaneously with Siegert's theorem and with the empirical data of the $\gamma^{*} N \rightarrow \Delta(1232)$ quadrupole form factors.

We conclude first that the relations (3)-(4) implies that 
Siegert's theorem is violated by terms $\mathcal{R}_{p t}=\mathcal{O}\left(1 / N_{c}^{2}\right)$, which may be a sizable error in the case $N_{c}=3$. Since the relations (31)-(4) are extrapolated from large $N_{c}$ they can have relative deviations of the order $1 / N_{c}^{2}$. We then use the constraints of Siegert's theorem to modify the relation for $G_{E}$. We obtain parametrizations for the quadrupole form factors that violate Siegert's theorem only by terms $\mathcal{R}_{p t}=\mathcal{O}\left(1 / N_{c}^{4}\right)$. This result is thus compatible with Siegert's theorem apart from higher-order corrections in $1 / N_{c}^{2}$.

We look also for additional contributions for the transition form factors $G_{E}$ and $G_{C}$, namely the contributions from the valence quarks from the nucleon and $\Delta(1232)$ systems. As mentioned, those contributions are small in the context of quark models but combined with the parametrizations of the pion cloud contributions they can reduce the gap between theory and data. An interesting propriety of the valence quark contributions for the electromagnetic form factors is that they vanish in the pseudo-threshold limit, as consequence of the orthogonality between the nucleon and $\Delta(1232)$ wave functions. As a consequence, the test of Siegert's theorem condition $\mathcal{R}_{p t}=0$ needs to be tested only for the pion cloud contribution of the transitions form factors.

At the end, we combine valence and pion cloud contributions using a model compatible with Siegert's theorem apart from higher-order corrections in $1 / N_{c}^{2}$. The results are then compared with the empirical data for $G_{E}$ and $G_{C}$, showing a fair description of the overall data.

\section{PION CLOUD CONTRIBUTIONS}

We can test the quality of the relations (3)-(44) comparing those functions with the data based on some parametrization for $G_{E n}$. To represent the electric form factor of the neutron, we considers the Galster parametrization 22]

$$
G_{E n}\left(Q^{2}\right)=-\mu_{n} \frac{a \tau_{N}}{1+d \tau_{N}} G_{D}
$$

where $\mu_{n}=-1.913$ is the neutron magnetic moment, $\tau_{N}=\frac{Q^{2}}{4 M^{2}}, G_{D}=1 /\left(1+Q^{2} / 0.71\right)^{2}$ is the dipole factor, and $a, d$ are two dimensionless parameters.

The quadrupole form factors obtained with the parameters $a=0.9$ and $d=2.8$ [11] are presented in Fig. 1] For a better test of Siegert's theorem we multiply the function $G_{C}$ and the data for $G_{C}$ by $\kappa$. The calculations are compared with the data from Mainz [13], MIT-Bates [23] and Jefferson Lab 24] for finite square momentum transfer, $Q^{2}$, and the world average from the Particle Data Group for $Q^{2}=0$ [25]. The data are compiled in Ref. [26].

It is clear in Fig. 1 that, the difference between the parametrizations for $G_{E}$ and $\kappa G_{C}$ is not zero in the pseudo-threshold limit, when $Q^{2} \simeq-0.1 \mathrm{GeV}^{2}$. This result implies that Siegert's theorem is violated, because $\mathcal{R}_{p t} \neq 0$. The explicit calculation of the deviation using

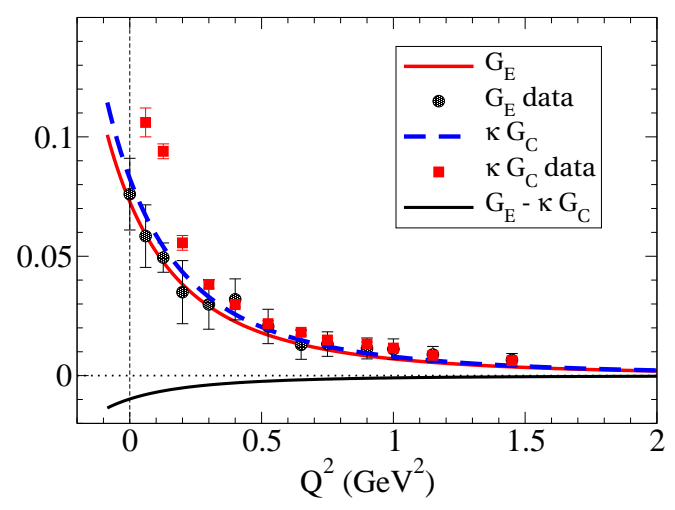

FIG. 1: $\gamma^{*} N \rightarrow \Delta$ quadrupole form factors estimated by the pion cloud parametrization of Eqs. [3)- (4). Data from Ref. [26].

$G_{E n}\left(Q_{p t}^{2}\right) \simeq-\frac{1}{6} r_{n}^{2} Q_{p t}^{2}$, gives

$$
\mathcal{R}_{p t} \simeq-\left(\frac{M}{M_{\Delta}}\right)^{3 / 2} \frac{r_{n}^{2}}{12 \sqrt{2}} Q_{p t}^{2} .
$$

Since $Q_{p t}^{2}=-\left(M_{\Delta}-M\right)^{2}$ and $M_{\Delta}-M=\mathcal{O}\left(1 / N_{c}\right)$ we can conclude that $\mathcal{R}_{p t}=\mathcal{O}\left(1 / N_{c}^{2}\right)$. Although a result $\mathcal{O}\left(1 / N_{c}^{2}\right)$ may be seen as a small quantity, the numerical value is still sizable, as we can see in the graph for $\mathcal{R}=$ $G_{E}-\kappa G_{C}$ at the pseudo-threshold $\left(\mathcal{R}_{p t}\right)$.

\section{VALENCE QUARK CONTRIBUTIONS}

Before discussing how to improve the pion cloud parametrization of the quadrupole form factors $G_{E}$ and $G_{C}$, we may question if Siegert's theorem can in fact be verified for the valence quark sector.

We look then for the results obtained within valence quark models. We consider, in particular, the covariant spectator quark model developed in Refs. 33, 16, 2729] for the nucleon and $\Delta(1232)$ systems. The basic assumptions of the model are that: (i) in the electromagnetic interaction the photon couples with the single quark (impulse approximation) while the other two quarks can be interpreted as an effective diquark, (ii) the quarks have their own internal structure (dressed by gluons and quark-antiquark states), and (iii) the radial quark-diquark wave functions are calibrated in terms of momentum range parameters that can be estimated by physical or lattice QCD data.

Concerning the nucleon and $\Delta(1232)$ systems the model is quite successful in the description of the data. The parameters associated with the quark structure (quark electromagnetic form factors) were first fixed by the nucleon elastic form factor data [28]. After that the model was used to estimate the valence quark contribution for the $\gamma^{*} N \rightarrow \Delta$ magnetic dipole form factor [27]. The results are compatible simultaneously with estimates from dynamical reactions models [15] and with lattice QCD simulations [3, 16, 27, 30]. The model for 


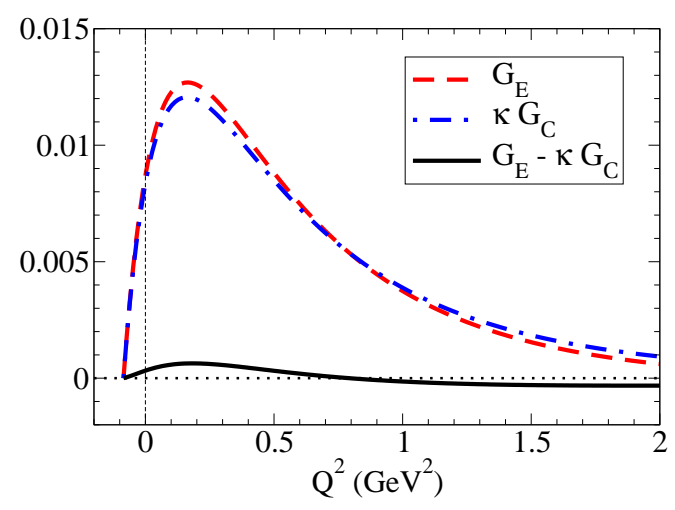

FIG. 2: Valence quark contributions for the $\gamma^{*} N \rightarrow \Delta$ quadrupole form factors estimated by the covariant spectator quark model [16].

the $\Delta(1232)$ was then extended with the inclusion of $D$ states in the wave function [3, 16].

We consider, in particular, the parametrization from Ref. [16]. In that work the $\Delta(1232)$ system is described by a combination a $S$-wave and two $D$-wave quarkdiquark states. The $D$ states can be labeled as $D 3$ (quark total spin 3/2) and $D 1$ (quark total spin 1/2) [3, 27]. The nucleon system is represented just by a $S$ state 28 . In the limit where the $D$-state mixtures vanish, we obtain $G_{E} \equiv 0$ and $G_{C} \equiv 0$. The $D$-state mixtures and the $D$-radial wave functions are determined by a fit to the lattice QCD data from Ref. [31] and then extrapolated to the physical point. The admixture of $D$ states is about $0.72 \%$ for both states. The extrapolation from lattice QCD for the physical regime is performed using a vector meson parametrization of the quark current (quark electromagnetic form factors). More details can be found in Refs. [16, 29, 30].

The valence quark contributions to the quadrupole form factors $G_{E}$ and $G_{C}$ from Ref. [16] are presented in Fig. 2. In the figure, we again compare $G_{E}$ with $\kappa G_{C}$. The more interesting aspect of the figure is that Siegert's theorem is exactly verified, as we can see from the result $\mathcal{R}=G_{E}-\kappa G_{C}=0$ at the pseudo-threshold. This result is a consequence of $G_{E}=G_{C}=0$ at the same point.

In the covariant spectator quark model, the quadrupole form factors $G_{E}$ and $G_{C}$ are calculated in terms of angular integrals of a function $b(k, q)$, where $k$ and $q$ are, respectively, the diquark and the photon momenta. In the pseudo-threshold limit $(|\mathbf{q}|=0)$, the function $b(k, q)$ reduces to the spherical harmonic $Y_{20}(\hat{\mathbf{k}})$ [3]. The presence of the function $Y_{20}(\hat{\mathbf{k}})$ is then the consequence of the overlap between the $\Delta(1232) D$ states and the nucleon $S$ state. Since in the pseudo-threshold limit there is no dependence in the photon momentum $|\mathbf{q}|$, the angular integrals are reduced to the angular integration of $Y_{20}(\hat{\mathbf{k}})$, which vanishes. Consequently the form factors vanish too. The result $G_{E}=G_{C}=0$ at the pseudo-threshold is then the corollary of the orthogonality between $S$ and $D$ states.

In Fig. 2, we can notice the turning of the functions
$G_{E}$ and $G_{C}$ near $Q^{2}=0.15 \mathrm{GeV}^{2}$, just above the photon point $Q^{2}=0$ and a soft convergence to zero at the pseudo-threshold. The reduction of the quadrupole form factors near $Q^{2}=0$ can also be seen in the lattice QCD data 16, 31]. As we will see next, the presence of the maximum for $G_{C}$ near $Q^{2}=0.15 \mathrm{GeV}^{2}$, instead at $Q^{2}=0$, has implications in the values of $G_{C}$ at small $Q^{2}$.

Another interesting aspect concerning Fig. 2 is the function $\mathcal{R}=G_{E}-\kappa G_{C}$ for finite $Q^{2}$. We can see that the function $\mathcal{R}$ is very small compared with $G_{E}$ or $\kappa G_{C}$. We then concludes, that in the covariant spectator quark model, the results $G_{E}$ and $\kappa G_{C}$ are very similar. It is possible that the relation $G_{E} \simeq \kappa G_{C}$ is also valid for other quark models. We note, in particular, that the estimate of the quark core contribution used in the SatoLee model assumes $G_{E}=\kappa \frac{M_{\Delta}+M}{2 M_{\Delta}} G_{C} \simeq \kappa G_{C}$ [15].

\section{SIEGERT'S THEOREM}

We can now discuss if, as for the valence quark sector, the pion cloud parametrization is also consistent with Siegert's theorem.

In the large $N_{c}$ limit the baryons are infinitely heavy and can be treated as static. For this reason large $N_{c}$ is mostly used to calculate static proprieties of the baryon and transition between baryon states [32, 33]. In particular, the relations (3)-(4) are extensions of large $N_{c}$ relations for $G_{E}$ and $G_{C}$ at $Q^{2}=0$ for finite $Q^{2}$ [7]. Those relations are not derived explicitly from large $N_{c}$, but, since they derivation for $Q^{2}=0$ is based on large $N_{c}$, one can infer that they are also limited by the accuracy from the large $N_{c}$ analysis, and can therefore be affected by relative corrections of the order $1 / N_{c}^{2}[7,[9,11,112,32]$.

Instead of using the $1 / N_{c}$ expansion to calculate possible $1 / N_{c}^{2}$ relative corrections, one can use Siegert's theorem to check if there are corrections that are consistent with the theorem. One notes that the use of constraints external to the $S U(6)$ and large $N_{c}$ formalisms were used already in the calculation of coefficients associated with physical quantities as the charge radius, quadrupole moments and others [12, 34 36.

In a first attempt we checked if we can have an exact description of Siegert's theorem, modifying the functions $G_{E}$ or $G_{C}$ keeping at the same time the results for $Q^{2}=$ 0 , derived from large $N_{c}$. Considering the replacement $G_{E} \rightarrow\left(1+\alpha Q^{2}\right) G_{E}$, we can preserve the result from large $N_{c}$ at $Q^{2}=0$, if we calculate $\alpha$ in order to obtain $\mathcal{R}_{p t}=0$ at the pseudo-threshold. The solution for this condition is $\alpha=-\frac{1}{M_{\Delta}^{2}-M^{2}}$. We obtain the same effect if we replace $G_{C} \rightarrow G_{C} /\left(1+\alpha Q^{2}\right)$. The problem of the new form for $G_{E}$ is that it vanishes when $Q^{2}=M_{\Delta}^{2}-M^{2}$, in conflict with the data.

We then try an approximated solution, replacing $G_{E} \rightarrow G_{E} /\left(1+Q^{2} /\left(M_{\Delta}^{2}-M^{2}\right)\right)$, which induces no zeros 
for $Q^{2}>0$. One obtain in this case

$$
G_{E}\left(Q^{2}\right)=\left(\frac{M}{M_{\Delta}}\right)^{3 / 2} \frac{M_{\Delta}^{2}-M^{2}}{2 \sqrt{2}} \frac{\tilde{G}_{E n}\left(Q^{2}\right)}{1+\frac{Q^{2}}{M_{\Delta}^{2}-M^{2}}} .
$$

The previous relation differs from Eq. (3) at the pseudothreshold only by a term $\mathcal{O}\left(1 / N_{c}^{2}\right)$.

With the new form for $G_{E}$, one obtains

$$
\mathcal{R}_{p t} \simeq\left(\frac{M}{M_{\Delta}}\right)^{3 / 2} \frac{M_{\Delta}-M}{2 M} \frac{r_{n}^{2}}{12 \sqrt{2}} Q_{p t}^{2}
$$

which is now a term $\mathcal{O}\left(1 / N_{c}^{4}\right)$, since $M_{\Delta}-M=\mathcal{O}\left(1 / N_{c}\right)$ and $M=\mathcal{O}\left(N_{c}\right)$.

The expected falloff for large $Q^{2}$ of the pion cloud contributions for the form factors $G_{E}$ and $G_{C}$ given by Eqs. (41) and (77) are $G_{E} \propto 1 / Q^{8}$ and $G_{C} \propto 1 / Q^{6}$ respectively. One recall however those contributions are derived from the low $Q^{2}$ expansion of the neutron electric form factor and its application is in principle limited to intermediate values of $Q^{2}$ [7]. The high $Q^{2}$ region is expected to be dominated by the valence quark degrees of freedom [37] as discussed later.

To summarize, we use Siegert's theorem to find a correction for the form factor $G_{E}$ that minimizes the violation of Siegert's theorem. The solution proposed, given by Eq. (77) is not exact, but reduces the violation of Siegert's theorem to a term of the order $1 / N_{c}^{4}$, The proposed function preserves the result for $G_{E}(0)$ obtained in the large $N_{c}$ framework, and correspond to a relative correction of $1 / N_{c}^{2}$ to the result of $G_{E}$ at the pseudothreshold.

\section{COMBINATION OF PION CLOUD AND VALENCE QUARK CONTRIBUTIONS}

Since, as discussed, the pion cloud component represents only the leading order effect in $G_{E}$ and $G_{C}$, we combine the new pion cloud parametrizations with the valence quark contributions of the covariant spectator quark model (consistent with Siegert's theorem). The sum of the two contributions is presented in Fig. 3 .

From Fig. 3, we conclude that, apart from the results for $G_{C}$ below $0.2 \mathrm{GeV}^{2}$, to be discussed later, we obtain a very good description of the overall data. This represents a considerable improvement comparative to the previous pion cloud parametrizations (see Fig. 11). In addition, the form factors are now compatible with Siegert's theorem, within an error of the order $1 / N_{c}^{4}$. The smallness of the error can be visualized in the figure if we look for $\mathcal{R}=$ $G_{E}-\kappa G_{C}$ at the pseudo-threshold.

For future reference we call the attention for the fact that, the nonzero results for the form factors $G_{E}$ and $G_{C}$ are a direct consequence of the pion cloud contributions, since as discussed, the valence quark contribution vanishes at the pseudo-threshold.

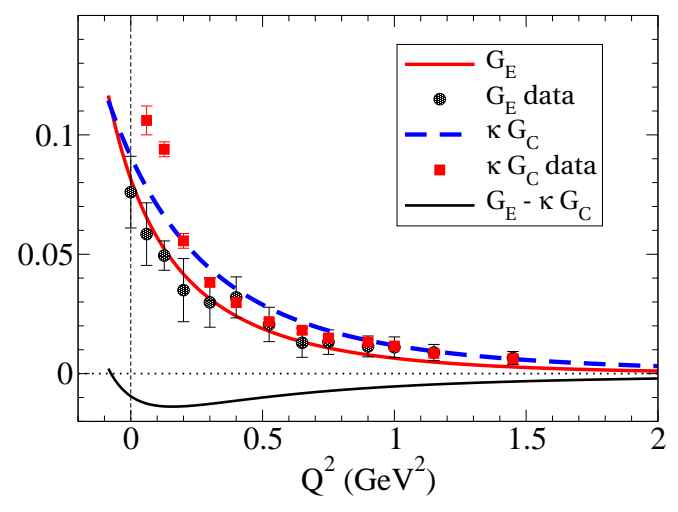

FIG. 3: $\gamma^{*} N \rightarrow \Delta$ quadrupole form factors estimated as a combination of the valence quark and pion cloud contributions. Data from Ref. [26].

For a final discussion of the results, we need to take into account that the pion cloud contributions for $G_{E}$ and $G_{C}$ can have relative deviations of the order $1 / N_{c}^{2}$ from Eqs. (4) and (7), as discussed previously. To represent those deviations we include a band of $\pm 10 \%$ in the graphs for $G_{E}$ and $\kappa G_{C}$ to represent the possible relative deviation (term of order $1 / N_{c}^{2}$ ) from the estimate of the pion cloud contribution. Note that the $\pm 10 \%$ deviation is more relevant for the discussion of the spacelike region, where the data are available, than near the pseudo-threshold. The final results for $G_{E}$ and $G_{C}$ including the variation band are presented in Fig. 4. In the figure we compare also the results with the MAID-SG2 parametrization from Ref. [19], in order to better visualize the difference between our model and the data at low $Q^{2}$. The MAID-SG2 gives a high quality description of the data, and it is compatible with Siegert's theorem.

From the graph for $G_{C}$, we can conclude that, the gap between the present model and the data for $Q^{2}<0.2$ $\mathrm{GeV}^{2}$ may not be explained by the pion cloud contribution, since the data are out of the band. We tested unsuccessfully if the quality of the description at low $Q^{2}$ could be improved considering a parametrization of the $G_{E n}$ data more complex than the Galster parametrization (5). Those results are an indication that the gap between model and data may be a consequence of the valence quark contributions. Parametrizations of the quark core contributions closer to a dipole form as in the Sato-Lee and DMT models from Ref. [15, 17] are more appropriate to describe the data measured at low $Q^{2}$. Those parametrizations, however, differ in shape, from the estimates presented in Fig. 2, and are not compatible with the shape required by Siegert's theorem and the soft convergence to zero at the pseudo-threshold. A comparison between the results from the covariant spectator quark model and the parametrization from Ref. 15] can be found in Ref. [16]. As discussed in the context of the covariant spectator quark model, the convergence to zero at the pseudo-threshold is a consequence of the orthogonality between the nucleon and the $\Delta(1232)$ states. 

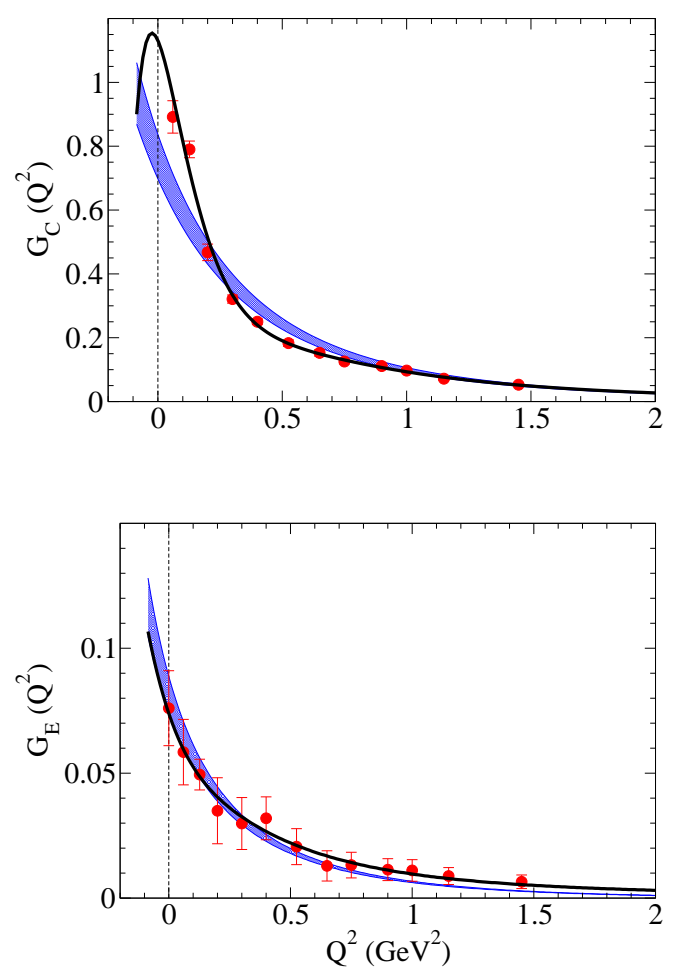

FIG. 4: Results for the $\gamma^{*} N \rightarrow \Delta$ quadrupole form factors in comparison with the MAID-SG2 parametrization from Ref. [19]. The bands indicate an estimate of the pion cloud error of $10 \%$, equivalent to the order of $1 / N_{c}^{2}$. Data from Ref. [26].

The shape of the valence quark contributions in the region $Q^{2}=0-0.2 \mathrm{GeV}^{2}$ can in principle be tested with the help of lattice QCD simulations. With the advent of the lattice QCD simulations near the physical point, it is expected that in the near future the lattice QCD simulations approaches the physical point. In those conditions, lattice simulations in quenched QCD and partially quenched QCD approximations may be compared with our estimate of the valence quark contributions. Also, the pion cloud contributions can be estimated from the comparison between full QCD and quenched QCD. An indication that the low $Q^{2}$ data for $G_{C}$ are reliable comes from the chiral effective-field theory, which connects the lattice QCD data with large pion masses with the physical regime [38].

Independent of the source of the discrepancy of $G_{C}$ for $Q^{2}<0.2 \mathrm{GeV}^{2}$, the shape of the form factor $G_{C}$ has implications in the Coulomb quadrupole square radius. Some authors argue that the low $Q^{2}$ behavior of $G_{C}$ is a consequence of the long extension of the pion cloud [1]. Other works suggest instead, that, as a consequence of Siegert's theorem, $G_{C}$ becomes smoother near $Q^{2}=0$ [19], which according with the present analysis may be a consequence of the valence quark contributions, since the pion cloud contribution is sharper near $Q^{2}=0$.

In the range of the data presented $Q^{2}<2 \mathrm{GeV}^{2}$ the pion cloud contributions are still sizable (see Fig. 1). As discussed the pion cloud parametrization are in principle valid for low $Q^{2}$ and may be modified for larger values of $Q^{2}$. For $G_{E}$ the valence quark component falls as $1 / Q^{4}$, as expected from a quark model [3, 16], and dominates over the pion cloud component $\left(1 / Q^{6}\right)$. As for $G_{C}$ the valence quark component falls as $1 / Q^{6}$, as the pion cloud component. The final falloff is then $G_{E} \propto 1 / Q^{4}$ and $G_{C} \propto 1 / Q^{6}$, respectively, consistent with pQCD [37]. We conclude then that, for very large $Q^{2}$, the present model is compatible with the expected pQCD falloff. Recall that the pQCD falloff is the consequence of the dominance of the valence quark contributions [37]. Another pQCD prediction is that, $G_{E} \simeq-G_{M}$, for very large $Q^{2}$ [37, 39]. Experimentally we are nowadays far away from this result [4, 37].

Overall, our calculations support the idea that the physics associated with the $\gamma^{*} N \rightarrow \Delta(1232)$ transition can be described by adding pionic degrees of freedom to the quark models [4, 40, 41]. Dynamical reaction models such as the Sato-Lee model [15] and the DMT model [17, 18], which calculate the pion cloud dynamically, are also in qualitative agreement with the data. In those models the bare core contributions are estimated phenomenologically as already discussed for the case of Ref. [15].

\section{SUMMARY AND CONCLUSIONS}

In conclusion, we have proposed a new pion cloud parametrization for the $\gamma^{*} N \rightarrow \Delta(1232)$ electric quadrupole form factor. The new form for $G_{E}$ is consistent with Siegert's theorem, $G_{E}=\kappa G_{C}$, at the pseudothreshold, within an error of $1 / N_{c}^{4}$.

We have also discussed the implications of Siegert's theorem to the bare core contribution of the quadrupole form factors. Contrary to the pion cloud contributions, the valence quark contributions vanish at the pseudothreshold.

Combining the new parametrizations of pion cloud contributions with the valence quark contributions for the same form factors, we have obtained a very accurate description of the quadrupole form factors data, apart from a small underestimation of the $G_{C}$ data in the region $Q^{2}=0-0.15 \mathrm{GeV}^{2}$.

Future developments in lattice QCD may help to clarify if the the gap between theory and data at low $Q^{2}$ is a consequence of the underestimation of the valence quark component or of the pion cloud component.

\section{Acknowledgments}

The author thanks João Pacheco B. C. de Melo and Kazuo Tsushima for the hospitality at Universidade Cruzeiro do Sul, where this work started, and Pulak Giri for useful suggestions. This work is supported by the Brazilian Ministry of Science, Technology and Innovation and by the project "Science without Borders" from 
the Conselho Nacional de Desenvolvimento Científico e
Tecnológico (CNPq) 400826/2014-3.
[1] H. F. Jones and M. D. Scadron, Annals Phys. 81, 1 (1973).

[2] V. Pascalutsa, M. Vanderhaeghen and S. N. Yang, Phys. Rept. 437, 125 (2007) hep-ph/0609004.

[3] G. Ramalho, M. T. Peña and F. Gross, Phys. Rev. D 78, 114017 (2008) arXiv:0810.4126 [hep-ph]].

[4] I. G. Aznauryan et al., Int. J. Mod. Phys. E 22, 1330015 (2013) arXiv:1212.4891 [nucl-th]].

[5] G. Ramalho, M. T. Peña and A. Stadler, Phys. Rev. D 86, 093022 (2012) arXiv:1207.4392 [nucl-th]].

[6] G. Ramalho, M. T. Peña and F. Gross, Phys. Lett. B 678, 355 (2009) arXiv:0902.4212 [hep-ph]]. G. Ramalho, M. T. Pena and F. Gross, Phys. Rev. D 81, 113011 (2010) arXiv:1002.4170 [hep-ph]].

[7] V. Pascalutsa and M. Vanderhaeghen, Phys. Rev. D 76, 111501 (2007) arXiv:0711.0147 [hep-ph]].

[8] A. J. Buchmann, E. Hernandez and A. Faessler, Phys. Rev. C 55, 448 (1997) nucl-th/9610040.

[9] P. Grabmayr and A. J. Buchmann, Phys. Rev. Lett. 86, 2237 (2001) hep-ph/0104203.

[10] A. J. Buchmann, Phys. Rev. Lett. 93, 212301 (2004) hep-ph/0412421.

[11] A. J. Buchmann, Can. J. Phys. 87, 773 (2009) arXiv:0910.4747 [physics.atom-ph]].

[12] A. J. Buchmann, J. A. Hester and R. F. Lebed, Phys. Rev. D 66, 056002 (2002) hep-ph/0205108.

[13] S. Stave et al. [A1 Collaboration], Phys. Rev. C 78, 025209 (2008) arXiv:0803.2476 [hep-ex]].

[14] S. Capstick and G. Karl, Phys. Rev. D 41, 2767 (1990).

[15] B. Julia-Diaz, T.-S. H. Lee, T. Sato and L. C. Smith, Phys. Rev. C 75, 015205 (2007) nucl-th/0611033.

[16] G. Ramalho and M. T. Peña, Phys. Rev. D 80, 013008 (2009) arXiv:0901.4310 [hep-ph]].

[17] S. S. Kamalov and S. N. Yang, Phys. Rev. Lett. 83, 4494 (1999) nucl-th/9904072.

[18] D. Drechsel, S. S. Kamalov and L. Tiator, Eur. Phys. J. A 34, 69 (2007) arXiv:0710.0306 [nucl-th]].

[19] G. Ramalho, Phys. Rev. D 93, 113012 (2016) arXiv:1602.03832 [hep-ph]].

[20] G. Ramalho, Phys. Lett. B 759, 126 (2016) arXiv:1602.03444 [hep-ph]].

[21] A. J. Buchmann, E. Hernandez, U. Meyer, and A. Faessler, Phys. Rev. C 58, 2478 (1998).

[22] S. Galster, H. Klein, J. Moritz, K. H. Schmidt, D. We- gener and J. Bleckwenn, Nucl. Phys. B 32, 221 (1971).

[23] N. F. Sparveris et al. [OOPS Collaboration], Phys. Rev. Lett. 94, 022003 (2005) nucl-ex/0408003.

[24] J. J. Kelly et al., Phys. Rev. C 75, 025201 (2007) nucl-ex/0509004]; I. G. Aznauryan et al. [CLAS Collaboration], Phys. Rev. C 80, 055203 (2009) arXiv:0909.2349 [nucl-ex]].

[25] K. A. Olive et al. [Particle Data Group Collaboration], Chin. Phys. C 38, 090001 (2014).

[26] V. I. Mokeev, https://userweb.jlab.org/ mokeev/ resonance_electrocouplings/

[27] G. Ramalho, M. T. Peña and F. Gross, Eur. Phys. J. A 36, 329 (2008) arXiv:0803.3034 [hep-ph]].

[28] F. Gross, G. Ramalho and M. T. Peña, Phys. Rev. C 77, 015202 (2008) nucl-th/0606029.

[29] G. Ramalho, K. Tsushima and F. Gross, Phys. Rev. D 80, 033004 (2009) arXiv:0907.1060 [hep-ph]].

[30] G. Ramalho and M. T. Peña, J. Phys. G 36, 115011 (2009) arXiv:0812.0187 [hep-ph]].

[31] C. Alexandrou, G. Koutsou, H. Neff, J. W. Negele, W. Schroers and A. Tsapalis, Phys. Rev. D 77, 085012 (2008) arXiv:0710.4621 [hep-lat]].

[32] E. E. Jenkins, X. Ji and A. V. Manohar, Phys. Rev. Lett. 89, 242001 (2002) hep-ph/0207092.

[33] R. F. Dashen, E. E. Jenkins and A. V. Manohar, Phys. Rev. D 49, 4713 (1994) Erratum: [Phys. Rev. D 51, 2489 (1995)] hep-ph/9310379.

[34] G. Dillon and G. Morpurgo, Phys. Lett. B 448, 107 (1999).

[35] A. J. Buchmann and R. F. Lebed, Phys. Rev. D 62, 096005 (2000) hep-ph/0003167.

[36] A. J. Buchmann and E. M. Henley, Phys. Rev. D 65, 073017 (2002).

[37] C. E. Carlson and N. C. Mukhopadhyay, Phys. Rev. Lett. 81, 2646 (1998) hep-ph/9804356.

[38] V. Pascalutsa and M. Vanderhaeghen, Phys. Rev. Lett. 95, 232001 (2005) hep-ph/0508060.

[39] C. E. Carlson, Phys. Rev. D 34, 2704 (1986).

[40] M. Fiolhais, B. Golli and S. Širca, Phys. Lett. B 373, 229 (1996).

[41] D. H. Lu, A. W. Thomas and A. G. Williams, Phys. Rev. C 55, 3108 (1997). 